\title{
Préface : Les nouveaux visages de la performativité
}

Preface: The New Faces of Performativity

Jérôme Denis

\section{(2) OpenEdition}

\section{Journals}

Édition électronique

URL : http://journals.openedition.org/edc/344

DOI : $10.4000 /$ edc.344

ISSN : 2101-0366

Éditeur

Université de Lille

\section{Édition imprimée}

Date de publication : 1 décembre 2006

Pagination : 8-24

ISBN : 2-9514961-7-6

ISSN : $1270-6841$

\section{Référence électronique}

Jérôme Denis, "Préface : Les nouveaux visages de la performativité ", Études de communication [En ligne], 29 | 2006, mis en ligne le 20 novembre 2014, consulté le 21 décembre 2020. URL : http:// journals.openedition.org/edc/344; DOI : https://doi.org/10.4000/edc.344

Ce document a été généré automatiquement le 21 décembre 2020.

(C) Tous droits réservés 


\title{
Préface : Les nouveaux visages de la performativité
}

\author{
Preface: The New Faces of Performativity
}

\author{
Jérôme Denis
}

1 Ces dernières années, la notion de performativité a eu un certain succès dans les sciences sociales. Ce succès a participé de ce que certains désignent comme un double tournant épistémologique. D'un côté, un tournant linguistique qui doit beaucoup aux relectures du "second» Wittgenstein (1961) et qui a vu naître un intérêt majeur pour les pratiques langagières et plus généralement la " communication » dans des sphères qui en était étrangères (par exemple l'étude des pratiques scientifiques, celle des activités juridiques, ou encore des sciences de l'organisation). De l'autre, un tournant pratique (Schatzki et al., 2001) qui a suscité de nombreuses recherches attachées à décrire et à comprendre l'action et les activités qui composent leur objet de recherche. Ces deux mouvements ont amené certains à mettre au centre de leur questionnement non seulement la part langagière de l'action, mais aussi la nature pragmatique du langage, c'est-à-dire les actes de langage eux-mêmes. Dans ce déplacement, la notion de performativité a été extraite de son cadre initial (la philosophie du langage ordinaire, puis la linguistique) pour être confrontée à des objets et des traditions disciplinaires très différents. C'est à la problématisation de certaines de ces confrontations que le présent numéro d'Études de communication propose de s'atteler. À l'opposé d'une utilisation comme "faire-valoir" de la notion initialement forgée par J.-L. Austin (1970), les articles rassemblés ici cherchent à mettre en évidence les intérêts concrets et parfois les limites qu'elle représente. Ils proposent ainsi un panorama interdisciplinaire ${ }^{1}$ qui fait de ce numéro un espace ouvert au débat pour questionner la notion, sa portée heuristique et les transformations que chacun opère pour en faire un instrument solide dans l'élaboration d'une problématique partagée, à la croisée de la communication et de l'action.

2 En guise d'introduction, je tenterai de rassembler les différentes propositions empiriques et théoriques qui sont faites au fil des articles en montrant, malgré leur hétérogénéité, qu'elles participent de près ou de loin à un mouvement plus vaste de 
réappropriation des premières intuitions de J.-L.Austin, que lui-même abandonne assez tôt dans ses conférences. Je questionnerai dans un premier temps les modalités de ce qui semble être, à la lumière des conclusions austiniennes, un retour en arrière. J'essaierai de montrer qu'il est étroitement lié au type d'objet de recherche qui sont réinvestis à l'aune de la performativité. Dans un second temps, j'entrerai dans le détail de cette reconquête théorique qui, a bien des égards, aboutit à une redéfinition en profondeur.

\section{Les migrations d'une notion}

3 La notion de performativité est un cas intéressant pour qui aime étudier la manière dont les concepts scientifiques circulent entre des auteurs, des objets de recherche, voire des espaces disciplinaires. Alors que, dans le même recueil qui en pose les premières définitions, J.-L. Austin l'abandonne (ou en tout cas montre la nécessité de la dépasser), elle est restée vivace chez d'autres. Comme nous le verrons dans certains textes de ce numéro, c'est même parfois « contre » Austin, dans une critique plus ou moins virulente de sa philosophie du langage ordinaire, que la notion est ravivée. Il n'est bien sûr pas question ici de prétendre retracer exhaustivement la manière dont elle s'est disséminée. Cependant, on peut repérer dans le mouvement migratoire représenté en partie par les articles de ce numéro, les ingrédients d'un débat plus vaste entre linguistique et sciences sociales. Dans la confrontation de la question performative à de nouveaux objets, ce sont les définitions mêmes du langage, de l'action et de leurs relations qui sont en jeu.

\section{De la performativité à la valeur illocutoire et retour}

4 Le projet de J.-L.Austin (1970) est bien connu: en voulant définir le caractère performatif de certains énoncés, il cherche à spécifier les différentes occasions où l'énonciation n'est pas en train de "constater " une action ou une situation, mais constitue elle-même une action à part entière. En étudiant ainsi les cas où «dire c'est faire ", il rompt avec une immense partie de la philosophie du langage traditionnelle. Mais au fil de son ouvrage, les choses se compliquent. À l'issue de la septième conférence, il fait finalement un constat d'échec du programme qu'il s'était fixé. Distinguer performatif et constatif est loin d'être si simple que le laissaient penser les premiers mouvements de sa réflexion. Les «malheurs » d'abord ne sont pas totalement réservés aux énonciations performatives, tout comme l'exigence de conformité factuelle n'est pas exclusive aux constatifs. Aucun critère grammatical ne lui a par ailleurs permis de distinguer les énonciations performatives. Enfin, le caractère « explicite » de certaines énonciations qui se montrent performatives ne suffit pas pour les classer à coup sûr dans les performatifs. Qui plus est, il les fait finalement tomber sous le coup d'une épreuve que l'on croyait définitivement éloignée : celle qui tranche entre le vrai et le faux.

Une fois ce constat fait, J.-L. Austin délaisse la question de la performativité en tant que qualité " pure » attribuée à des énonciations spécifiques, pour « reprendre le problème à neuf " et travailler à la mise en lumière de l'épaisseur pragmatique de toute énonciation, qu'il décompose en actes locutoires (qui ont une signification), illocutoires (qui ont une force) et perlocutoires (qui ont des effets). Ce faisant, il assume 
explicitement le passage d'une problématique de la distinction entre performatifs et constatifs à une théorie des actes de discours (p.152) qu'il peaufine dans la dernière conférence en dressant une liste des valeurs illocutoires de l'énonciation.

Volontiers associées à la nébuleuse philosophique qui a nourri le tournant pragmatique des sciences sociales, les réflexions de

J.-L. Austin n'ont pas été suivies jusqu'au bout par tous les chercheurs qui s'en réclament. C'est au nom même de l'intérêt pour les pratiques ordinaires, de la nécessité de documenter des situations concrètes (et non de courts exemples de conversation simulée) que certains ont fait machine arrière dans le mouvement analytique de J.L. Austin en redonnant à la notion de performativité une spécificité, une force heuristique, qu'ils refusent de confondre avec celle du concept de force illocutoire ${ }^{2}$. Si toute énonciation suppose un acte illocutoire, toutes ne sont pas performatives dans le sens à la fois restreint et élargi qui s'élabore au fil des analyses dont les textes de ce numéro témoignent. Et c'est par l'enquête ethnographique, soucieuse des conditions effectives de leur réalisation que ces énonciations performatives peuvent être analysées à leur juste valeur.

Dans ce retour à la performativité, on peut voir un nouveau jeu d'équilibre entre langage, action et situation. Ce qui est finalement reproché aux conclusions de J.L. Austin, c'est le « tout linguistique » auxquelles elles mènent, finissant par négliger la part située des actes de discours. La reprise de la notion de performativité constitue ainsi une sorte d'hétérodoxie qui, sauvant la notion, cherche à remettre en avant le rôle des conventions, des institutions et plus généralement des « conditions de félicité » qui la composaient initialement (Fraenkel, 2006). Cette posture hétérodoxe doit beaucoup aux célèbres critiques que P. Bourdieu a adressées à J.-L. Austin (1982), insistant sur la nécessité de prendre en compte dans l'analyse des performatifs la position des énonciateurs dans l'espace social et le type de pouvoir dont ils disposent. Elle s'en éloigne pourtant en partie, se renouvelant aujourd'hui aux frontières de la sociolinguistique, de la sociologie et des sciences de la communication. Elle permet notamment d'élargir l'éventail des exemples canoniques de J.-L. Austin pour confronter la notion à des objets variés, appréhendés en situation réelle. Chacun de ces objets est l'occasion de construire sur elle un regard aiguisé, invitant à en redessiner les contours.

\section{Nouveaux objets, nouveaux regards}

8 Encore une fois, il n'est pas question ici de prétendre à une quelconque exhaustivité. Les usages de la notion de performativité sont si nombreux qu'il faudrait sans doute un article entier pour seulement en citer les références. On peut toutefois proposer de reconstituer une «famille » de pensée, interdisciplinaire, à laquelle appartiendrait les articles présentés dans ce numéro.

9 Dans cette famille, la plus connue et la plus facilement repérable est sans doute J. Butler $(1988$; 2004) qui a profondément marqué le champ des gender studies et plus largement des cultural studies en y intégrant une réflexion fondée sur la notion de performativité. Attachée à comprendre le "pouvoir des mots » (notamment des insultes), elle montre l'enjeu politique fort de leur manipulation et leur appropriation. Comme nous le verrons, cette version de la performativité connaît aujourd'hui de nombreuses critiques. Certains y voient un culturalisme exacerbé qui passe à côté de l'épaisseur pratique des énonciations performatives. 
10 D'autres recherches se sont focalisé sur un aspect plus institutionnel de la performativité. C'est le cas notamment du vaste courant d'analyse en sciences de l'information et de la communication qui examine les actes de langage au sein des organisations (Taylor, Van Every, 2000 ; Gramaccia, 2001). Dans cette perspective, les organisations ne sont jamais des entités "données ", identifiées une bonne fois pour toute: elle sont "performées» par d'innombrables échanges et transactions quotidiennes. Ce courant est représenté dans ce numéro par un article de B. Fauré et G. Gramaccia. Dans d'autres termes, plus politiques, on retrouve cet intérêt pour le travail ordinaire de construction du réel dans l'article de P. Gonzalez qui montre comment la notion de performativité peut servir l'ethnologie du phénomène religieux telle qu'elle est pratiquée par É. Claverie (2003) et A. Piette (2003).

11 Les analyses des performativités scientifique et juridique se préoccupent elles aussi de performativité institutionnelle, mais à un autre niveau. Dans la mouvance des travaux de la théorie de l'acteur-réseau (Latour, 2006), c'est un véritable "programme performatif » qui s'est constitué, trouvant sa source dans un ouvrage coordonné par M. Callon sur la science économique (Callon, 1998b) et prochainement approfondi dans un nouvel ouvrage collectif (MacKenzie et al., à paraître). Ce programme, qui a suscité d'innombrables débats, vise à montrer comment la théorie économique (economy) performe en partie l'économie pratique (economics). Ce faisant, il reprend une problématique déjà travaillée en marge (ou en prémices) de l'économie des conventions, par exemple par L. Boltanski (1979) à propos de la catégorie statistique des « cadres » ou encore par A. Desrosières (1993). Mais il y ajoute un intérêt particulier pour les dispositifs techniques qui décuplent la force performative des énoncés théoriques et des formules ${ }^{3}$. C'est donc à un double objet qu'est confrontée ici la notion de performativité: la science, mais aussi les innovations technologiques qu'elle participe à élaborer. C'est sur ce second objet que se focalise J.-T. Julia dans ce numéro à propos des interactions dites "homme/machine». En s'appuyant sur le modèle de l'analyse relationnelle des fonctions illocutoires de l'école de Genève (Roulet et al., 2001), il cherche à montrer qu'au-delà d'une performation pure de l'échange par des dispositifs médiatiques, on peut repérer des «incomplétudes interactionnelles » qui complexifient les transactions entre l'utilisateur et la machine.

12 Enfin, on compte parmi les cinq articles de ce numéro, deux études consacrées au droit. On le sait, ce domaine d'activité est au centre de nombreux exemples de J.-L. Austin. Il est donc difficile de considérer que s'intéresser à la performativité juridique constitue un déplacement majeur pour les usages de la notion. Et pourtant, comme nous le verrons, c'est sans doute dans le réinvestissement du droit lui-même que l'on trouve les critiques les plus frontales des développements de J.-L. Austin. Dans son analyse d'une série d'auditions à distance, équipées de dispositifs de visiophonie, C. Licoppe remet en question l'immédiateté performative des énoncés canoniques d'ouverture et de fermeture de séances. Il alimente dans le même temps le débat sur l'équipement technologique de la performativité. B. Fraenkel investit quant à elle le droit comme un lieu doublement stratégique pour refonder la notion même de performativité. Elle explique d'abord que le droit constitue une véritable matrice pour la pensée de J.-L. Austin (qui a travaillé en étroite collaboration avec H.-L. Hart) et que la performativité qu'il cherche à définir est pour grande partie une performativité juridique, sans que cela soit toutefois explicitement revendiqué par lui. Enfin, et surtout, elle montre que les analyses de J.-L. Austin s'appuient implicitement, et sans 
jamais la problématiser, sur la mise en équivalence d'actes de langage oraux et écrits. C'est en approfondissant les différences fondamentales entre les deux et en montrant que le droit se nourrit essentiellement des seconds qu'elle élabore un modèle de la performativité écrite.

13 On le voit, parmi ces nombreux projets, certains revendiquent une ambition théorique qui ne s'appuie sur la notion de performativité qu'au prix de profondes transformations. C'est moins l'apport de la notion aux situations étudiées qui importe que le nouvel éclairage de la première par les secondes.

\section{Activités, matérialité, temporalité : une performativité repensée}

14 La nécessité de repenser en profondeur la performativité est inextricable d'une certaine posture ethnographique qui transforme radicalement l'analyse des « conditions de félicité ». Saisies sur le vif, celles-ci ne sont plus figées sous la forme de procédures et de principes conventionnels stabilisés qu'il suffirait de respecter pour réussir. Elles sont appréhendées en tant que situations concrètes d'accomplissement des énonciations performatives. Désormais situées, les «conditions de félicité » sont affaire de circulation dans un espace et surtout un temps qui débordent largement l'ici et maintenant de la parole. Ce n'est qu'une fois ce profond déplacement mis en lumière qu'il est possible de rendre compte des dimensions qui composent une nouvelle définition de la performativité.

\section{Par-delà les conditions de félicité}

15 Comment caractériser les modalités d'accomplissement des performatifs? Deux dimensions sont centrales dans les analyses présentées ici et dans le mouvement de réappropriation plus général dont elles témoignent: la nature collective du travail nécessaire à l'élaboration des énoncés performatifs et la matérialité des éléments sur lesquels s'appuie - et que produit - ce travail.

16 J.-L. Austin lui-même évoque régulièrement le fait que les énoncés performatifs ne peuvent pas être tenus par un être solitaire. Pour être heureux, ils nécessitent un auditoire qui non seulement est "performé » par les énoncés (en devenant mariés par exemple), mais participe pleinement à cette performation en tant qu'ingrédient productif de certaines conditions de félicité (en consentant, en se passant les bagues, etc.). Cependant la focalisation de J.-L. Austin sur les énoncés eux-mêmes l'empêche d'accepter, ou en tout cas d'étudier de front, la part résolument collective de leur accomplissement. Dans la veine de P. Grice (1972), P. Gonzalez approfondit ici cette voie en montrant comment l'«institution du divin » dans la prédication évangélique est affaire de "montage collaboratif ». C'est par les actions combinées du prédicateur et des fidèles présents que réussit la situation de prédication. C'est aussi ce que montre C. Licoppe en s'inspirant des multiples recherches du courant de l'analyse conversationnelle ${ }^{4}$ qui ont mis en lumière la part coopérative et procédurale de ce qu'il faudrait appeler l'interaction performative. Par un examen minutieux de situations d'audience, il interroge la place qu'y tiennent des énoncés qui «ouvrent» et «ferment» la séance, tenus pour inamovibles et individuels dans le modèle de la performativité. Cette analyse lui permet de souligner l'aspect processuel et graduel des 
ouvertures et des fermetures d'audiences effectuées à distance. Il donne ainsi à voir une véritable " activité performative » qui se déploie bien avant et bien après l'énoncé luimême, jusqu'à parfois s'en passer.

Il existe une autre manière d'insister sur la nature collective de la performativité, qui déplace le regard vers les instances énonciatrices. Comme le montre B. Fraenkel dans ce numéro, il y a une véritable incapacité pour J.-L. Austin (et beaucoup d'autres à sa suite) à prendre en compte cet aspect. Cela doit beaucoup à son refus de considérer l'écriture et la lecture comme relevant d'une forme spécifique de performativité. Mais dès lors que l'on sort des situations canoniques et dématérialisées de paroles déclamées, la pluralité des entités énonciatrices devient flagrante. L'analyse que fait B. Fraenkel des actes juridiques souligne à quel point tant les activités d'inscription et de réinscription que les pratiques de lecture et de relecture engagent de nombreux acteurs qui tous participent à des degrés plus ou moins fort à l'acte performatif. Dans un autre article, elle montre qu'observer les modalités de fabrication et de circulation des écrits juridiques permet de comprendre leur nature composite et elle souligne à quel point l'énonciation performative est à la fois résultat et source d'un engagement collectif. Ainsi, dans le cas des huissiers de Justice, la signature elle-même, acte performatif par excellence que l'on pourrait juger purement individuel, « engage[-t-il] tout à la fois l'huissier et l'étude en tant que lieu de travail équipé, des personnes et des machines » (Fraenkel, 2006, p. 111) $)^{5}$. L'énonciation performative est distribuée.

Lorsqu'ils mettent en lumière la manière dont les faits scientifiques sont établis, circulent, et participent à la performation du monde qu'ils décrivent, B. Latour (1989), M. Callon (1998a) ou encore D. MacKenzie (2003) tirent les mêmes conclusions quant à la nature collective de la performativité (et plus indirectement au rôle de l'écrit). Pour qu'ils deviennent de "véritables » performatifs, les faits, les théories ou les formules doivent circuler dans des chaînes de traduction qui consolident l'assemblage des entités qui le composent et leur permet d'acquérir le statut de «matters of fact ». Cette circulation nécessite un travail collectif sans lequel ce qui est performé se délite et finit par disparaître (Latour, 2006) ${ }^{6}$. C'est aussi, mais d'un autre point de vue, ce que souligne M. Lenglet dans ce numéro lorsqu'il propose de réinvestir de front les pratiques discursives qui ont cours dans le monde de la finance. Pour que les normes soient respectées et les engagements tenus, les prestataires de services d'investissement ont développé une «fonction déontologique » qui opère comme un véritable médiateur de performativité.

19 La dimension matérielle de la performativité est l'autre élément central de ces analyses. Elle est notamment un moyen d'éviter le risque du «tout linguistique » que représente la focalisation sur la force illocutoire des énoncés, sans toutefois basculer dans une perspective qui ne serait que culturaliste, faisant reposer la performativité sur des conventions abstraites et arbitraires ou une "culture" jamais incarnée. Ici encore, l'intérêt heuristique des enquêtes sur les pratiques d'écriture est indéniable, notamment parce qu'elles portent avec elles le souci de penser le rôle des supports, des formes et des matières. Ainsi le panneau, le registre ou l'écran sont-ils des ingrédients essentiels à la performativité. Comme le précise très clairement B. Fraenkel dans son texte : l'interdiction d'entrée ne vaut comme avertissement que si et seulement si elle est affichée, c'est-à-dire inscrite sur un objet qui peut être attaché au lieu et donc inséré dans l'environnement ${ }^{7}$. La force performative ne tient pas dans la seule forme 
grammaticale de l'énoncé, ni dans la situation d'énonciation : elle doit beaucoup aux caractéristiques formelles et matérielles des objets qui le façonnent.

Dans un article qui fait le point sur le programme performatif en sociologie économique, M. Callon généralise cette préoccupation matérielle (Callon, à paraître). Il montre que la "performation" de la pratique (economics) par la théorie (economy) s'opère dans un jeu d'ajustements mutuel entre des « agencements sociotechniques » et les énoncés. Insister sur ces agencements, et donc rappeler encore l'intérêt de la théorie de l'acteur-réseau pour les objets techniques, est selon M. Callon essentiel pour détacher le modèle de la performativité de celui de la performance au sens théâtral du terme et ses excès culturalistes. Il se range ainsi du côté de A. Mol lorsque celle-ci reproche à J. Butler de ne pas prendre en considération le corps et sa matérialité dans les ingrédients de la performation des identités et des genres. Pour performer son identité, le langage et les conventions « sociales" ne suffisent pas. Le corps lui-même doit être travaillé, "enacted», c'est-à-dire engagé d'une certaine manière dans des situations et des activités spécifiques.

Plus généralement, le renouvellement des approches des conditions de félicité soulève une question sensible, susceptible d'enrichir la critique des premières intuitions austiniennes. Car s'il inscrit au cœur de l'analyse la dimension située de toute énonciation performative, c'est finalement pour élargir une vision trop restrictive de la situation de profération telle que l'envisage J.-L. Austin. Distribuée entre différentes instances, la performativité telle qu'elle apparaît ici est aussi déployée dans le temps. Cette dimension est l'épicentre de la redéfinition que proposent certains auteurs.

\section{Les temps de la performativité}

B. Fraenkel fait de la question temporelle la pierre de touche des critiques qu'elle adresse à J.-L. Austin (et É. Benveniste). Elle montre dans son article que les situations de parole très limitées invoquées par Austin l'empêchent de saisir ce qui semble pourtant être l'essence de la performativité de l'énonciation juridique, sa capacité à durer. C'est la permanence, écrit-elle, qui caractérise «le mode de présence caractéristique du droit». L'écrit et ses différents supports en sont les principaux appuis : ils demeurent, circulent dans l'espace et le temps, et évitent que la force performative des énonciations se consume dans l'instant. Tirant toutes les conclusions de l'attention minutieuse qu'elle porte aux actes d'écriture, B. Fraenkel propose ainsi une redéfinition profonde de la performativité. Elle montre comment les actes juridiques produisent et mobilisent des énoncés à la " performativité latente » et écarte du même coup le présent de la situation comme seul et unique contexte (et condition) du performatif.

Que font ces énoncés « latents » et leur manipulation, sur le monde ? Ils en assurent la continuité et la solidité. On peut s'en saisir, les réactiver, faire appel à eux (moyennant un travail toujours collectif d'inscriptions, réinscriptions, lectures et relectures) pour que les choses reviennent à leur juste place. Et même lorsqu'il est question de créer une situation nouvelle (pour une déclaration de naissance par exemple), il s'agit avant tout de produire celle-ci légitimement, c'est-à-dire d'assurer son existence au sein d'un vaste corps de règles qui en définissent les contours et les conditions et lui permette d'être à son tour mobilisée dans de nouveaux actes juridiques. 

prédicateur évangélique, tels qu'ils sont produits dans la situation collective de la prédication, participent de l'instauration d'un réel, de son "institution». Cette institution s'opère à la croisée du constatif et du performatif. Comme le souligne l'auteur, cette "politique du réel », ancrée dans le texte biblique, est très proche de celle qu'opère la référence scientifique. Nous retrouvons ici les nombreux travaux de la théorie de l'acteur-réseau qui n'ont eu de cesse de montrer comment les faits étaient " construits ", non pas pour en critiquer le caractère arbitraire mais au contraire pour mettre en lumière les conditions concrètes de leur solidité. Dans son dernier ouvrage, B. Latour (2006) tente de clarifier le programme de ce courant théorique. Il souligne notamment les nombreux malentendus que le terme même de "construction " a pu engendrer. Revendiquant une posture relativiste à la manière des physiciens, il s'empare de la notion de performativité, retrouvant en les généralisant les analyses de M. Callon. Elle est pour lui le moyen de rendre compte du travail incessant des médiateurs (humains et non-humains) qui tissent le social quotidiennement (pp. 52-62). 
C'est dans un sens très proche que se situe l'analyse de ce que B. Fauré et G. Gramaccia appellent ici les «actes de calcul». Ces derniers participent en effet du processus langagier complexe qui consiste à faire exister quotidiennement une organisation. Ils participent de (et fondent parfois) l'ambiguïté des énoncés performatifs qui, comme dans les sphères religieuse et scientifique, tiennent lorsqu'ils réussissent à devenir des constatifs.

Qu'est-ce que performer alors? Ce n'est ni faire avec des éléments arbitraires tombés du ciel, ni reproduire des forces révélant un réel déjà là : c'est donner une forme, composer un monde commun'. On retrouve dans ce vocabulaire les préoccupations de G. Deleuze lorsqu'il oppose les couples de relations «virtuel/actuel» et «potentiel/ réel » (Deleuze, 1968). La performativité relève ici du régime de l'actualisation ${ }^{10}$. Dans les sciences de la vie, comme en économie, dans la religion ou en droit, cette actualisation s'opère via un travail complexe qui vise à produire des saillances. C'est ce qu'illustre M. Callon lorsqu'il déplace la question traditionnelle de l'encastrement des relations économiques dans les relations sociales. Il montre que la performativité des sciences économiques consiste précisément à isoler des processus "strictement économiques ", à les désencastrer pour ranger les relations sociales du côté des "externalités " (Callon, 1998a). On retrouve ce type d'analyse du côté de l'anthropologie de l'écriture. D. Pontille (2006), démarrant une réflexion sur la notion d'« acte ", met ainsi en lumière l'importance des formes de segmentation et de clôture qui caractérisent l'activité des huissiers de Justice : produire des listes exhaustives, désigner des choses, des personnes et des lieux dans des documents datés, signés et tamponnés, fermer et sceller des espaces. Comme la performation économique, l'institution du réel qu'accomplissent les actes juridiques prend la forme d'un travail de « circonscription» (p. 124).

Le second temps de l'analyse de P. Gonzalez pousse un peu plus loin cette réflexion. Dans la dernière partie de son texte, l'auteur souligne les risques inhérents à l'usage de la performativité comme mode de description et d'explication des phénomènes qu'il étudie. Sans entrer dans les détails, on retiendra parmi ces difficultés, celle qui confronte le chercheur aux catégories des personnes impliquées dans la situation qu'il examine. Il y a un risque en effet à proposer une définition hétérotopique du performatif qui présenterait des « auditeurs » subissant la performation plus qu'ils n'y participeraient. Ce risque est évité pour P. Gonzalez lorsque le chercheur est attentif à ce que lui «fait» le monde performé. C'est la résistance, aux yeux du chercheur extérieur, de ce monde dans lequel le prédicateur et ses auditeurs sont engagés ensemble qui donne à voir en quoi consiste la performativité évangélique. Il ne s'agit pas seulement de l'institution d'un réel par le prédicateur, mais du double mouvement de sa proposition et de son "habitation ", c'est-à-dire d'un engagement spécifique qui rend ce monde saillant, y compris à celui qui n'a pas les moyens de s'y engager.

Cet élément d'analyse pointe une deuxième dimension essentielle à la performativité telle qu'elle apparaît dans les courants exposés ici. Il souligne d'abord l'aspect définitivement situé de toute action performative. Aucune situation d'énonciation ne peut être considérée comme performative en soi. La performativité va toujours avec un monde spécifique qu'elle participe à instaurer ou à maintenir. Pour le dire en des termes wittgensteiniens, on ne peut jamais la détacher de la forme de vie dans laquelle elle se joue. Mais la question de l'engagement des personnes dans la situation de prédication fait aussi apparaitre les épreuves auxquelles tout énoncé qui prétend à la performativité doit faire face. Interrogée par les sciences de la communication, la 
sociologie, la gestion ou l'anthropologie, la réussite des énoncés performatifs n'est plus affaire de seules grammaire et conventions à respecter. Elle est conditionnée par des épreuves de réalité qui sont au fondement même de leur force. C'est parce qu'ils risquent d'échouer, et que leur échec aura des conséquences importantes, que des énoncés peuvent prétendre à la performativité. C'est aussi pour cette raison qu'ils sont complexes à élaborer, qu'ils nécessitent un travail particulier et qu'ils impliquent des collectifs hétérogènes. Les auteurs sont nombreux à insister sur ces épreuves. Sans l'engagement des fidèles, le divin n'est pas institué jusqu'au bout (Gonzalez, ce numéro) ; sans son attachement à des effets " garantis ", l'acte juridique ne tient pas (Fraenkel, ce numéro); sans l'attention à tous les détails, l'huissier peut produire un faux qui lui coûtera sa charge (Fraenkel, 2006), etc. Cela est aussi discuté en sociologie économique, notamment par D. MacKenzie (2003) qui se focalise sur la réussite ou non (il parle alors de " contre-performativité ») des théories à se traduire dans des dispositifs techniques.

La mise en avant d'un principe de réalité actualisé par des épreuves spécifiques et renouvelées sort définitivement le modèle de la performativité d'une vision strictement linguistique. De ce point de vue, une énonciation, ou plutôt une série d'énonciations et d'actions, ne sont performatives que si elles permettent de construire collectivement un monde qui tient face à différentes épreuves de réalité.

\section{Conclusion : pragmatique et politique}

La définition renouvelée de la notion de performativité n'est pas sans conséquence politique. Sous ces nouveaux visages, celle-ci apparait comme un moyen de lutter contre toute tentation naturaliste ou au contraire culturaliste. Dès lors que le caractère " performé » de certaines dimensions de notre réalité est mis en lumière, elles peuvent être à nouveau publiquement débattues. Mais ce débat est très différent de celui qui a été prôné par les tenants d'une pensée "critique ». Cette dernière vise essentiellement à remplacer le caractère "naturel » de l'objet critiqué par un caractère "social » ou « culturel » arbitraire, dans un mouvement quasi mécanique. À l'inverse, la critique que rend possible le nouveau programme performatif est profondément pluraliste. Elle consiste à remettre en question la nature des éléments qui ont été assemblés (et souvent l'assemblage lui-même), afin de montrer que d'autres mises en forme sont possibles. Mais ces nouvelles mises en forme ne pourront pas sortir du néant, ni être imposées d'elles-mêmes. Elles devront à leur tour être le fruit d'un long travail de performation: être discutées, expérimentées, autrement dit subir de nombreuses épreuves. Comprendre la part performative des activités qui constituent notre monde, c'est-à-dire comprendre en quoi elles sont en partie composées d'actes de langage situés, distribués et éprouvés, c'est ainsi refuser d'admettre aveuglément, et trop vite, les «naturalités» de toutes sortes: scientifiques, organisationnelles, économiques, juridiques... C'est laisser une place plus grande à l'incertitude, voire à l'indécision (Barthes, 2006), et défendre le pluralisme non pas seulement comme un puissant outil de description, mais aussi comme une voie politique possible. 


\section{BIBLIOGRAPHIE}

Austin, J. L., (1970), Quand dire c'est faire, Paris, Le Seuil.

Barthes, Y., (2006), Le pouvoir d'indécision. La mise en politique des déchets nucléaires, Paris, Economica.

Benveniste, É., (1976), Problèmes de linguistique générale, Paris, Galimard.

Boltanski, L., (1979), « Les systèmes de représentation d'un groupe social : les 'cadres' », Revue française de sociologie, Vol. 20, $\mathrm{n}^{\circ} 4$, pp. 631-667.

Bourdieu, P., (1982), Ce que parler veut dire. L'économie des échanges linguistiques, Paris, Fayard.

Butler, J., (1988), « Performative Acts and Gender Constitution : An Essay in Phenomenology and Feminist Theory », Theatre Journal, Vol. 40, $\mathrm{n}^{\circ}$ 4, pp. 519-531.

Butler, J., (2004), Le pouvoir des mots. Politique du performatif, Paris, Éditions Amsterdam.

Callon, M., (1998a), « An essay on framing and overflowing : economic externalities revisited by sociology ", in : Callon M. (dir.), The Laws of the Market, Oxford, Blackwell publishers, pp. 244-269.

Callon, M., (dir.), (1998b), The Laws of the Market, Oxford, Blackwell publishers.

Callon, M., (à paraître), « What does it mean to say that Economics is Permformative ?", in : MacKenzie D., Muniesa F. et Siu L. (dir.), Performing Economics : How Markets Are Constructed, New York, Princeton University Press.

Callon, M. et Muniesa, F., (2003), « Les marchés économiques comme dispositifs collectifs de calcul », Réseaux, Vol. 21, n 122, pp. 189-233.

Claverie, É., (2003), Les guerres de la Vierge. Une anthropologie des apparitions, Paris, Gallimard.

Deleuze, G., (1968), Différence et Répétition, Paris, P.U.F.

Desrosières, A., (1993), La politique des grands nombres. Histoire de la raison statistique, Paris, La Découverte.

Fraenkel, B., (2006), « Le moment de la signature dans le travail d'huissier de Justice : une performativité située ", in : Bidet A., Borzeix A., Pillon T., Rot G. et Vatin F. (dir.), Sociologie du travail et activité, Toulouse, Octares, pp. 101-126.

Goodwin, C. et Heritage, J., (1990), « Conversation Analysis », Annual Review of Anthropology, Vol. 19, pp. 283-307.

Gramaccia, G., (2001), Les actes de langage dans les organisations, Paris, L'Harmattan.

Grice, P., (1972), « Logique et conversation », Communications, $\mathrm{n}^{\circ} 30$.

Latour, B., (1989), La science en action, Paris, La découverte.

Latour, B., (1999), Politiques de la nature. Comment faire entrer les sciences en démocratie, Paris, La Découverte.

Latour, B., (2002a), Jubiler ou les tourments de la parole religieuse, Paris, Les empêcheurs de penser en rond.

Latour, B., (2002b), La fabrique du droit, Paris, La Découverte, Coll. « Armillaire ». 
Latour, B., (2006), Changer de société, refaire de la sociologie, Paris, La Découverte, Coll. « Armillaire ».

Mackenzie, D. et Millo, Y., (2003), « Construction d'un marché et performation théorique. Sociologie historique d'une Bourse de produits dérivés financiers ", Réseaux, Vol. 21, n 122, pp. 13-61.

Mackenzie, D., Muniesa, F. et Siu, L., (dir.), (à paraître), Performing Economics : How Markets Are Constructed, New York, Princeton University Press.

Piette, A., (2003), Le fait religieux. Une théorie de la religion ordinaire, Paris, Économica.

Pontille, D., (2006), « Produire des actes juridiques », in : Bidet A., Borzeix A., Pillon T., Rot G. et Vatin F. (dir.), Sociologie du travail et activité, Toulouse, Octares, pp. 113-126.

Roulet, E. et al., (2001), Un modèle et un instrument d'analyse de l'organisation du discours, Berne, Peter Lang, Coll. « Sciences pour la communication ».

Schatzki, T. R., Knorr-Cetina, K. et Von Savigny, E., (dir.), (2001), The Practice Turn in Contemporary Theory, London, Routledge.

Schegloff, E. A., (1991), « Conversation Analysis and Socially Shared Cognition », in : Resnick L. B., Levine J. M. et Teasley S. D. (dir.), Perspectives on Socially Shared Cognition, Washington, American Psychological Association, pp. 150-171.

Scollon, R. et Scollon, S. W., (2003), Discourses in place. Language in the material world, London, Routledge.

Searle, J. R., (1972), Les actes de langage, Paris, Hermann.

Taylor, J. R. et Van Every, E. J., (2000), The emergent organization : Communication as its site and surface, Mahwah, Lawrence Erlbaum Associates.

Wittgenstein, L., (1961), Investigations philosophiques, Paris, Gallimard.

\section{NOTES}

1. Pour six articles au total, cinq disciplines sont représentées : les sciences de l'information et de la communication, la gestion, la sociologie, l'ethnologie et l'anthropologie.

2. Ce mouvement a démarré très tôt, notamment avec É. Benveniste (1976) et J.-R. Searle (1972) qui ont plaidé pour réserver la notion de performativité à un usage restreint.

3. Ce point a tout particulièrement été développé pour l'analyse des marchés financiers (Callon, Muniesa, 2003 ; MacKenzie, Millo, 2003).

4. Pour un panorama, voir C. Goodwin et J. Heritage (1990) et E. Schegloff (1991).

5. Nous verrons un peu plus tard que l'étude de ces modalités débouche sur une autre caractéristique des actes performatifs trop peu questionnée par Austin : leur temporalité.

6. Chez B. Latour cela déborde largement la science, comme ces récents travaux le montrent (Latour, 1999 ; 2002b ; a ; 2006). Si l'on prend le cas du mariage, sa perspective consisterait à suivre les mariés bien après la cérémonie pour repérer le travail sans cesse opéré par les nombreuses entités qui continuent à faire des deux protagonistes des mariés (citons sans chercher à aller plus loin les formulaires administratifs de tous ordres, le rappel de cet « état de fait » par les amis, la famille, et bien entendu le «travail » des alliances elles-mêmes pour soi et pour les autres). Sans ce travail l'état même de « marié » n'aurait plus ni sens, ni conséquence. 7. C'est l'objet central de la « géosémiotique » mise en œuvre par Scollon \& Scollon (2003). 
8. "[...] l'objet d'une définition performative disparaît dès qu'il cesse d'être 'performé' - ou s'il demeure, c'est que d'autres acteurs ont pris le relais " (Latour, 2006, p. 58).

9. Dans l'article de Gonzalez, comme chez Latour, ce n'est pas seulement un « réel » qui est institué (performé), mais dans un même mouvement les collectifs qui lui sont attachés.

10. «Performativity is not about creating, but about making happen » (Callon, à paraître).

\section{AUTEUR}

\section{JÉRÔME DENIS}

Département Sciences Économiques et Sociales - ENST 